\title{
Development of a Simple Fluorescence Sensing System for Carbonyl Stress in Urine
}

\author{
Wei-Jan Huang, ${ }^{\text {\# }}$ Yin-Tung Sun, ${ }^{2 \#}$ Wei-Han Lee, ${ }^{2}$ \\ Po-Yeh Lin, ${ }^{3}$ and Chien-Ming Chen ${ }^{4 *}$ \\ ${ }^{1}$ Graduate Institute of Pharmacognosy, Taipei Medical University, \\ No. 250, Wu-Hsing Street, Taipei 11031, Taiwan, ROC \\ ${ }^{2}$ Department of Mechanical Engineering, National Taipei University of Technology, \\ No. 1, Section 3, Zhongxiao East Road, Taipei 10608, Taiwan, ROC \\ ${ }^{3}$ School of Pharmacy, Taipei Medical University, \\ No. 250, Wu-Hsing Street, Taipei 11031, Taiwan, ROC \\ ${ }^{4}$ Department of Electro-Optical Engineering, National Taipei University of Technology, \\ No. 1, Section 3, Zhongxiao East Road, Taipei 10608, Taiwan, ROC
}

(Received July 6, 2021; accepted September 13, 2021)

Keywords: carbonyl stress, fluorescence, methylglyoxal, urine

In this study, we developed a simple fluorescence sensing system to rapidly measure the total carbonyl stress in urine because carbonyls may form advanced glycation end products, leading to diseases. The traditional method for detecting carbonyl stress has been high-performance liquid chromatography (HPLC), which detects glyoxal, methylglyoxal, and 3-deoxyglucosone contents. The excitation and emission wavelengths used in the sensing system to monitor aldehyde content in urine were 340 and $500 \mathrm{~nm}$, respectively. Rat urine samples were derivatized with 5,6-diamino-2,4-hydroxypyrimidine sulfate dihydrate. A $20 \mu \mathrm{L}$ sample was added to a carrier composed of polydimethylsiloxane for testing, and only $1 \mathrm{~s}$ was required for detection. According to the fluorescence intensity results, the calibration curve for the total content of all aldehydes in the urine had a good degree of linearity with the fluorescence intensity $\left(R^{2}=0.9879\right)$. In addition, the urine of diabetic and healthy rats was tested, and the correlation coefficient between the total carbonyl content detected by the simple fluorescence system and HPLC reached 0.8132. Collectively, our findings demonstrated that our system can rapidly measure total carbonyl stress in urine with a small sample volume.

\section{Introduction}

Carbonyl stress is caused by a series of active carbonyl compounds, including glyoxal, methylglyoxal, 3-deoxyglucosone, and acrolein. Oxidative pressure is generated mainly through free radical changes in proteins and lipids, which accumulate into carbonyl stress. ${ }^{(1)}$ When the concentration of carbonyls is very high, advanced glycation end products (AGEs) are formed. This process was originally discovered in the Maillard reaction. ${ }^{(2)}$ From a pathological viewpoint, AGEs can modify the biological structure or interact with specific cell receptors to

\#These authors contributed equally.

*Corresponding author: e-mail: cmchen@mail.ntut.edu.tw

https://doi.org/10.18494/SAM.2021.3533 
cause many diseases. ${ }^{(3,4)}$ Complications include diabetes, ${ }^{(5-7)}$ neurodegenerative diseases (Parkinson's disease and Alzheimer's disease), ${ }^{(8)}$ and male infertility. ${ }^{(9)}$

The most common method of detecting carbonyl stress is the detection of methylglyoxal, which has the highest carbonyl stress. ${ }^{(10)}$ High-performance liquid chromatography (HPLC) has been used to detect methylglyoxal. ${ }^{(1-14)}$ Although it takes a long time to detect a sample, it is still an important detection method. HPLC is highly accurate because it individually separates various compounds in the test substance.

When detecting methylglyoxal by the fluorescence method, methylglyoxal does not produce natural fluorescence by itself. Therefore, the fluorescent derivatization reagent 5,6-diamino-2,4hydroxypyrimidine sulfate dihydrate (DDP) is used to convert methylglyoxal to methyl-lumazine before detection. ${ }^{(14)}$

A simple fluorescence sensing system for D-lactate dehydrogenase detection was developed in 2015. ${ }^{(15)}$ In the present study, DDP was used to derivatize urine in accordance with a method proposed by Espinosa-Mansilla et al., ${ }^{(7)}$ and the total carbonyl stress in urine was directly measured using a fluorescence sensing system. HPLC was also used to determine the levels of single components, which were summed to obtain the carbonyl stress. Finally, we compared the results obtained using the two systems to explore their correlation.

\section{Materials and System Structure}

\subsection{Materials}

The miniature fiber-optic spectrometer (USB2000) used in this study was purchased from Ocean Optics (USA). A programmable DC power supply (LPS 305B-TC) was purchased from Motech Industries (Taiwan). An LED (UVA-340) was purchased from Roither Laser Technik GmbH (Vienna, Austria). Translation stages (TSDH-402S, TSDH-403L) and an optical lens (AGL-12-8.5p) were purchased from Sigma Koki (Tokyo, Japan). A FLUOstar OPTIMA microplate reader was purchased from BMG Labtech (Germany). Polydimethylsiloxane (PDMS) was purchased from SciKet (Taiwan). Citric acid monohydrate and sodium hydroxide were purchased from Nacalai Tesque (Kyoto, Japan). DDP was purchased from Tokyo Chemical Industry (Tokyo, Japan). Ammonium chloride and ammonia solutions were purchased from Kanto Chemical (Tokyo, Japan) and Showa Chemical (Tokyo, Japan), respectively. A methylglyoxal solution ( $40 \%$ in water) was obtained from Sigma-Aldrich (St. Louis, MO, USA). All solutions were prepared by dilution in ultrapure water obtained from a Milli-Q system. HPLC-grade acetonitrile (ACN) was obtained from Merck (Darmstadt, Germany).

\subsection{System structure}

The system structure was built using a $340 \mathrm{~nm}$ UV-LED as the excitation light, which can be focused on a sample carrier made of PDMS through an optical lens. The sample carrier was made into a V-shaped groove sheet. The PDMS spectrum is shown in Fig. 1. We selected PDMS as the material owing to its excellent inertness, light transmittance, and biocompatibility. This 


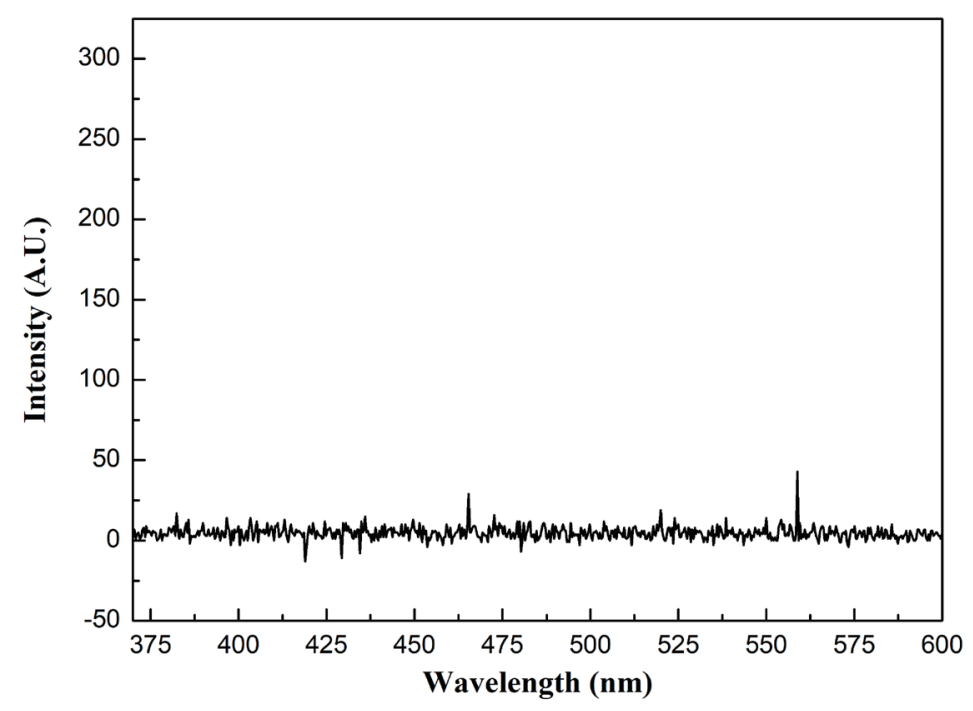

Fig. 1. Spectrum of sample carrier made of PDMS.

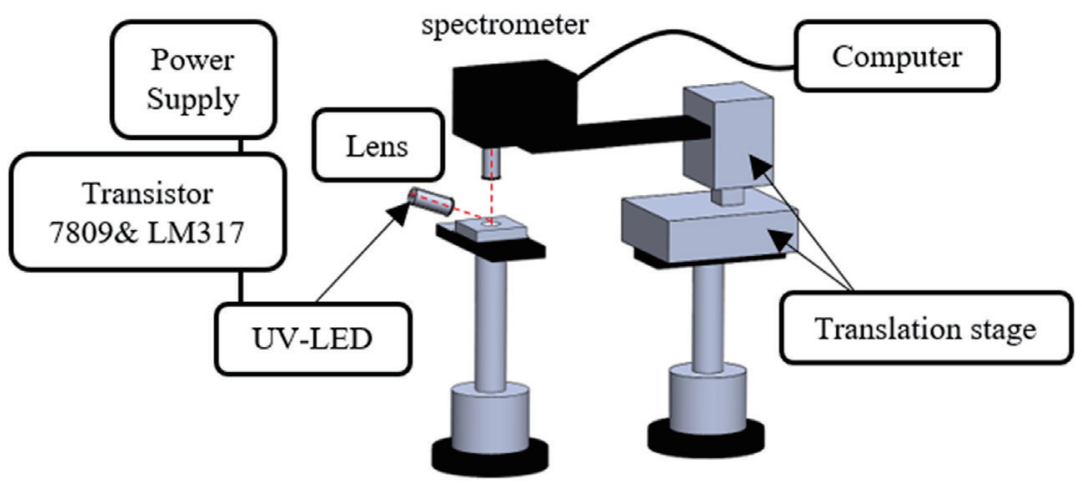

Fig. 2. (Color online) Architecture of proposed system.

method is suitable for detecting biological samples. To maintain the stable operation of the LED, a voltage-stabilizing circuit was fabricated with 7809 and LM317 transistors. The overall driving voltage was $7.0 \mathrm{~V}$ and the current was $10 \mathrm{~mA}$. The emission light from the sample passed through an optical lens and was focused on the USB2000 spectrometer, which was used as the receiving end of the system, and data were collected through a computer. All parts were built on a 3D translation stage to obtain the most suitable optical path. The architecture of this system is shown in Fig. 2.

\subsection{Derivatization}

The rat urine was shaken and centrifuged, and the middle part of the urine was collected for a series of concentration dilutions. We used ammonium chloride buffer $(\mathrm{pH} 10.0)$ and $7.5 \times 10^{-4} \mathrm{M}$ DDP, mixed them at a ratio of 2.5:50 $\mu \mathrm{L}$ (v:v) into an Eppendorf tube that was protected from light, and added $20 \mu \mathrm{L}$ of the prepared sample. The reaction mixture was incubated at $60{ }^{\circ} \mathrm{C}$ for 
$30 \mathrm{~min}$ and placed in an ice bath for $5 \mathrm{~min}$. Finally, $500 \mu \mathrm{L}$ of $0.01 \mathrm{M}$ citric acid buffer ( $\mathrm{pH}$ 5.88) was added to stop the derivatization reaction. Next, we took the diluted urine and added a series of concentrations of methylglyoxal standards and performed the same derivatization steps.

\section{Experimental Methods}

\subsection{Methylglyoxal standard product}

To confirm that the system could measure the carbonyl stress, we chose the methylglyoxal standard product, which had the highest proportion of carbonyl stress. The concentration was adjusted to $0,35,70,105,140,175,210,245,280,315,350,385,420,455,490,525$, and $560 \mu \mathrm{g} / \mathrm{L}$, and the fluorescence value for each concentration was recorded.

\subsection{Rat urine}

To confirm that rat urine could also be measured with this system, it was diluted to different concentrations, the fluorescence value was measured, and the accuracy and precision were analyzed. Rats were divided into diabetic and healthy control groups, and the analysis was performed at dilution of twofold and fourfold. The fluorescence value of urine was compared with the linearity of the methylglyoxal standard to determine the concentration of the diluted urine, and the corresponding concentration of the methylglyoxal standard was then added for recovery analysis.

\subsection{Comparison with HPLC}

Samples were randomly drawn from the urine of diabetic and healthy rats to determine the correlation between the total carbonyl stress measured by this simple fluorescence sensing system and the sum of each component measured by HPLC.

\section{Results and Discussion}

Figures 3 and 4 show that in the detection of methylglyoxal standard products, there is a good linear relationship between the sample concentration and the fluorescence. According to the measurements of the intra-assay and inter-assay, $R^{2}=0.9901$ and 0.9868 , respectively, the system can operate normally. Error bars represent the range of each measurement. The reasons for the error may include the concentration of DDP, the $\mathrm{pH}$ at the time of derivatization, and the temperature at the time of measurement. However, the error was generally within the allowable range.

The relationship between the fluorescence value and the dilution ratio of rat urine (1.25 to 32 times) is shown in Fig. 5. The fluorescence value had a linear relationship with the concentration $\left(R^{2}=0.9713\right)$. This shows that our system can measure methylglyoxal standard products and urine samples of rats with good linearity. 


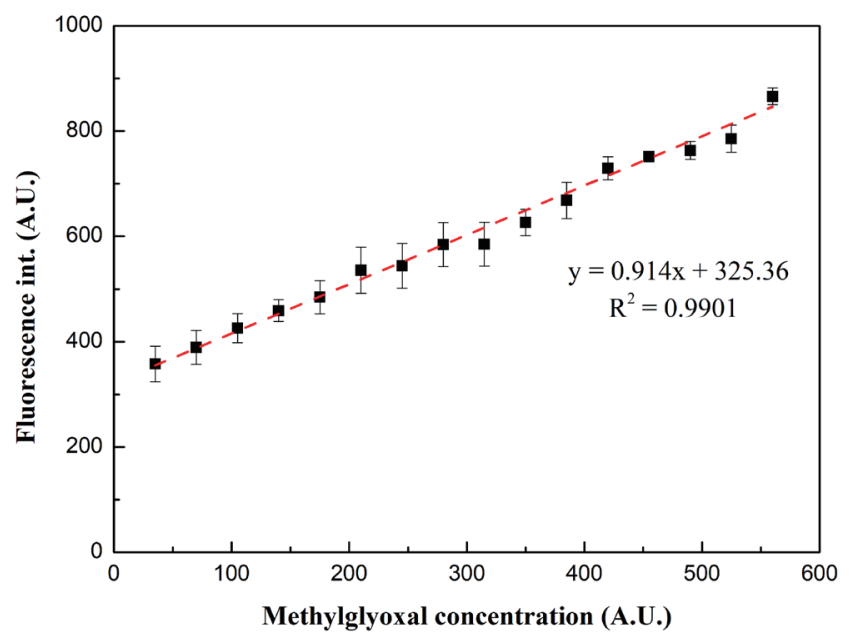

Fig. 3. (Color online) Calibration curve of methylglyoxal standard product for the intra-assay.

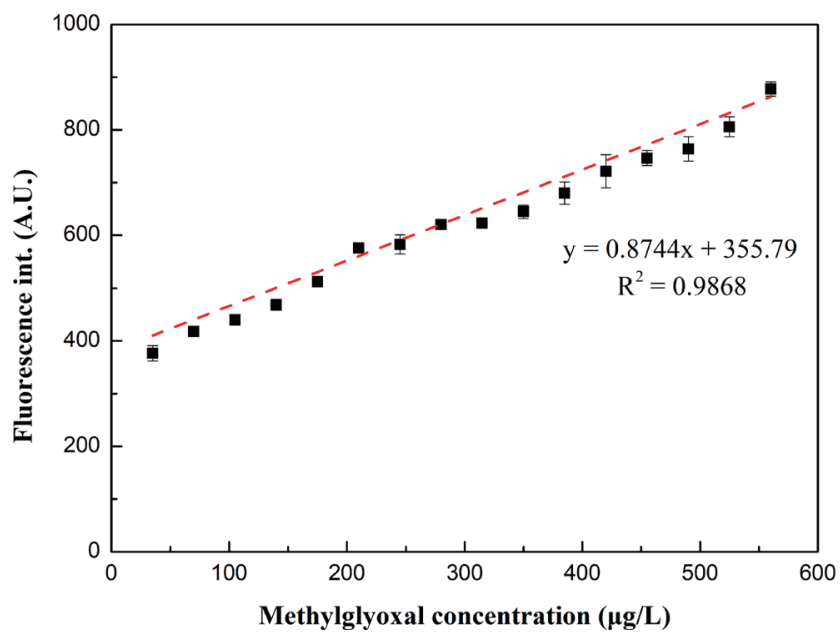

Fig. 4. (Color online) Calibration curve of methylglyoxal standard product for the inter-assay.

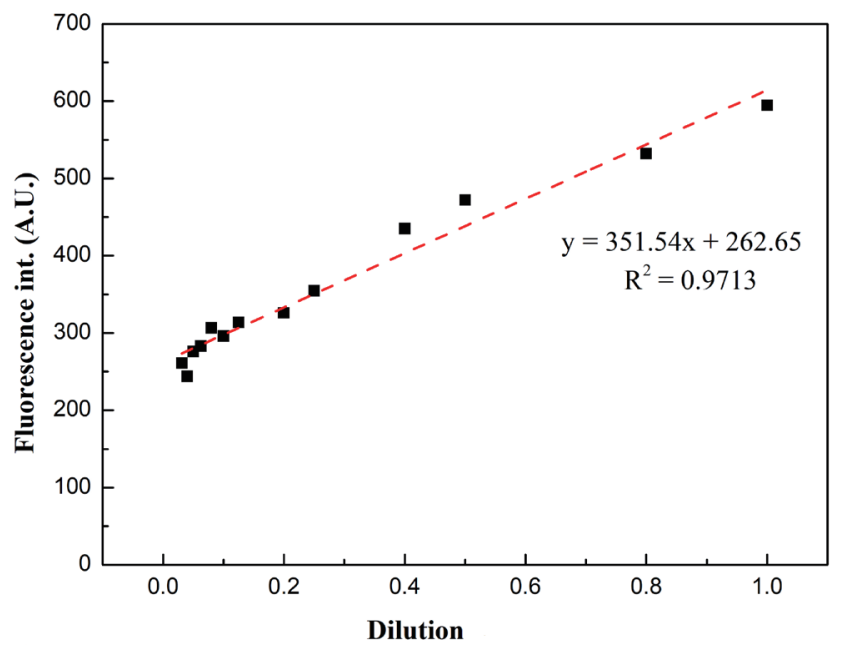

Fig. 5. (Color online) Calibration line of rat urine at different dilutions. 
The rat urine samples were diluted fourfold and twofold, and we compared the fluorescence value of each sample with the calibration curve of the methylglyoxal standard product to determine the concentration, and added a methylglyoxal standard solution of similar concentration for precision and accuracy analyses. The entire experimental procedure was performed using intra- and inter-assays, and the results are presented in Tables 1 and 2, respectively. "DM" and " $\mathrm{C}$ " in the tables represent diabetes and healthy control groups, respectively, and the number following them represents the dilution ratio. The calibration curve of the sample, which is for the urine of diabetic rats diluted twofold, is shown in Fig. 6 $\left(R^{2}=0.9879\right)$. The calculation results of the recovery rate were within the allowable error range, although a few larger errors mainly appeared in the rat urine diluted twofold, which might be

Table 1

Precision and accuracy analyses of intra-assay.

\begin{tabular}{lcccccc}
\hline \multirow{4}{*}{ DM4 } & Add MG $(\mu \mathrm{g} / \mathrm{L})$ & 0 & 105 & 122.5 & 140 & 157.5 \\
& Mean $(\mu \mathrm{g} / \mathrm{L})$ & 127.59 & 214.49 & 232.56 & 248.21 & 270.94 \\
& Recovery $(\%)$ & - & 82.76 & 85.69 & 86.16 & 91.02 \\
& RSD $(\%)$ & 6.27 & 5.19 & 6.29 & 6.6 & 7.2 \\
\hline \multirow{4}{*}{ DM2 } & Add MG $(\mu \mathrm{g} / \mathrm{L})$ & 0 & 210 & 227.5 & 245 & 262.5 \\
& Mean $(\mu \mathrm{g} / \mathrm{L})$ & 345.72 & 494.14 & 509.67 & 532.77 & 558.84 \\
& Recovery $(\%)$ & - & 70.68 & 72.07 & 76.35 & 81.19 \\
& RSD $(\%)$ & 1.57 & 4.17 & 2.23 & 2.03 & 1.86 \\
\hline \multirow{4}{*}{ C4 } & Add MG $(\mu \mathrm{g} / \mathrm{L})$ & 0 & 52.5 & 70 & 87.5 & 105 \\
& Mean $(\mu \mathrm{g} / \mathrm{L})$ & 72.6 & 128.65 & 149.06 & 165.72 & 175.69 \\
& Recovery $(\%)$ & - & 106.75 & 109.22 & 106.43 & 98.18 \\
& RSD $(\%)$ & 3.77 & 5.9 & 5.98 & 7.37 & 11.56 \\
\hline \multirow{2}{*}{ C2 } & Add MG $(\mu \mathrm{g} / \mathrm{L})$ & 0 & 140 & 157.5 & 175 & 192.5 \\
& Mean $(\mu \mathrm{g} / \mathrm{L})$ & 224.65 & 319.93 & 355.93 & 387.6 & 412.8 \\
& Recovery $(\%)$ & - & 68.48 & 83.73 & 93.46 & 98.05 \\
& RSD $(\%)$ & 3.11 & 0.39 & 2.19 & 2.83 & 1.67 \\
\hline
\end{tabular}

Table 2

Precision and accuracy analyses of inter-assay.

\begin{tabular}{lcccccc}
\hline \multirow{4}{*}{ DM4 } & Add MG $(\mu \mathrm{g} / \mathrm{L})$ & 0 & 105 & 122.5 & 140 & 157.5 \\
& Mean $(\mu \mathrm{g} / \mathrm{L})$ & 132.52 & 222.21 & 241 & 259.68 & 274.82 \\
& Recovery $(\%)$ & - & 85.42 & 88.56 & 90.83 & 90.35 \\
& RSD $(\%)$ & 3.48 & 2.9 & 3.14 & 2.41 & 1.6 \\
\hline \multirow{4}{*}{ DM2 } & Add MG $(\mu \mathrm{g} / \mathrm{L})$ & 0 & 210 & 227.5 & 245 & 262.5 \\
& Mean $(\mu \mathrm{g} / \mathrm{L})$ & 291.54 & 526.16 & 523.37 & 550.82 & 559.97 \\
& Recovery $(\%)$ & - & 111.72 & 101.9 & 105.83 & 102.26 \\
& RSD $(\%)$ & 2.82 & 2.5 & 1.6 & 0.86 & 2.04 \\
\hline \multirow{4}{*}{ C4 } & Add MG $(\mu \mathrm{g} / \mathrm{L})$ & 0 & 52.5 & 70 & 87.5 & 105 \\
& Mean $(\mu \mathrm{g} / \mathrm{L})$ & 81.39 & 125.17 & 141.83 & 177.23 & 184.31 \\
& Recovery $(\%)$ & - & 83.4 & 86.36 & 109.54 & 98.03 \\
& RSD $(\%)$ & 1.02 & 2.74 & 1.83 & 2.61 & 0.43 \\
\hline \multirow{4}{*}{ C2 } & Add MG $(\mu \mathrm{g} / \mathrm{L})$ & 0 & 140 & 157.5 & 175 & 192.5 \\
& Mean $(\mu \mathrm{g} / \mathrm{L})$ & 172.55 & 319.7 & 357.38 & 384.01 & 410.43 \\
& Recovery $(\%)$ & - & 105.1 & 117.35 & 120.83 & 123.57 \\
& RSD $(\%)$ & 0.39 & 1.51 & 1.13 & 2.11 & 0.99 \\
\hline
\end{tabular}




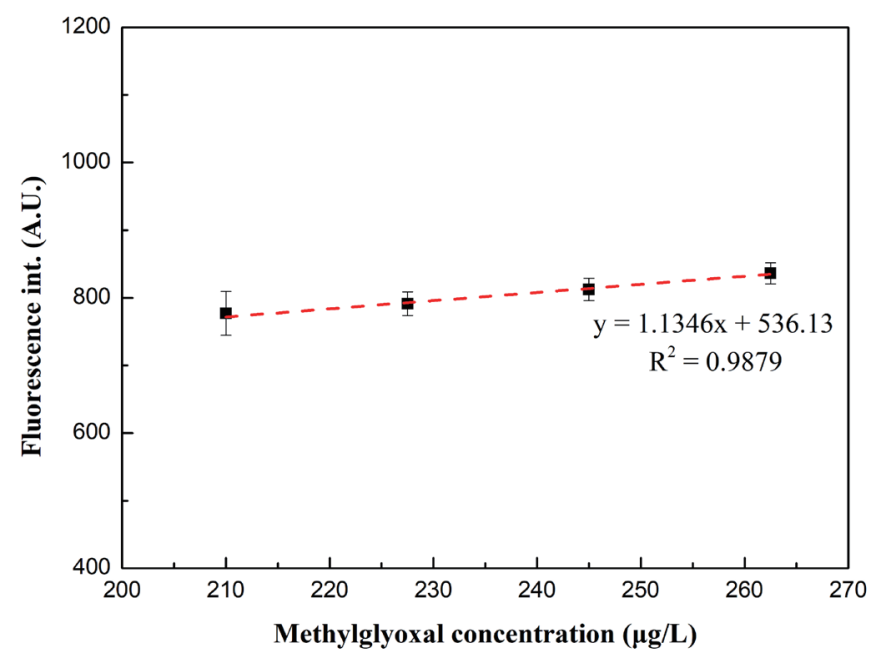

Fig. 6. (Color online) Calibration curve of urine diluted twofold.

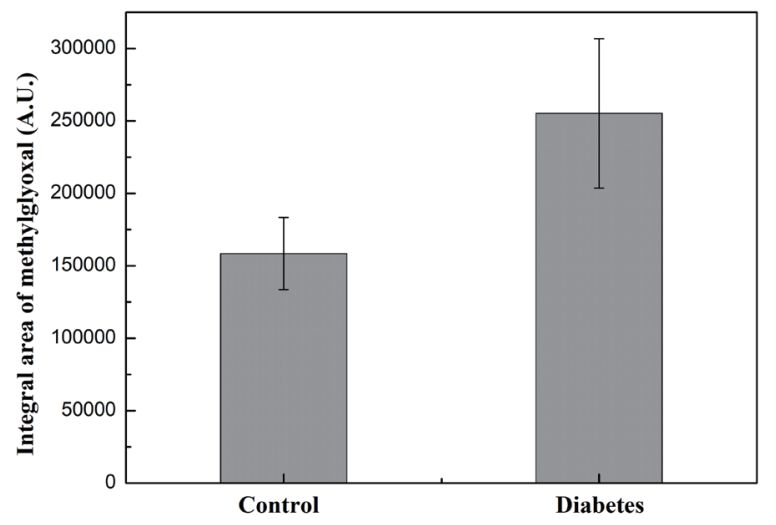

Fig. 7. Carbonyl stress detected in rat urine by HPLC.

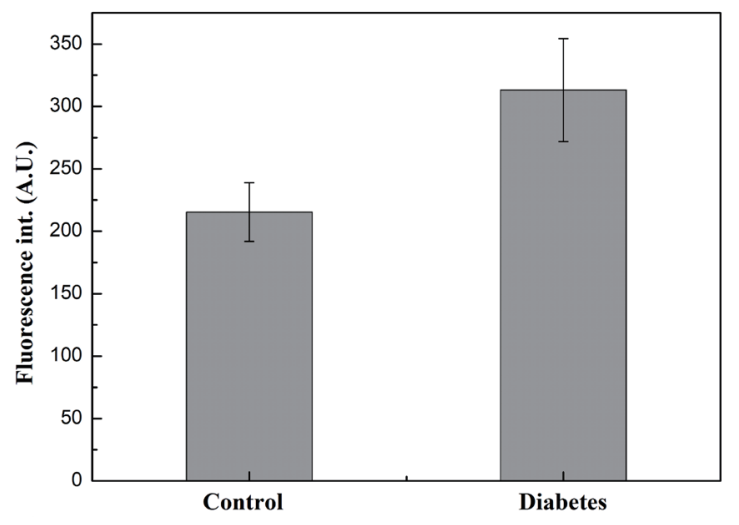

Fig. 8. Carbonyl stress detected in rat urine by fluorescence sensing system.

caused by impurities in the urine. Using this experiment as an example, rat urine diluted fourfold is a better choice. The relative standard deviation (RSD) is within the allowable error range.

Finally, several rat urine samples were diluted fourfold and derivatized using the same processing method. We compared the peak area values based on HPLC measurements, as shown in Fig. 7. The values based on the fluorescence intensity were measured by the fluorescence sensing system, as shown in Fig. 8. The values and standard deviations in the figure showed that the method we used could distinguish the urine of diabetic rats from that of healthy rats. We then compared the carbonyl stresses for HPLC and the present method, and obtained a Pearson correlation coefficient of $R^{2}=0.8132$, indicating a strong correlation.

This experiment preliminarily demonstrated that in contrast to HPLC, which required $75 \mathrm{~min}$ to detect the carbonyl stress in urine, our system required only $1 \mathrm{~s}$ for detection. Although there is a difference between the carbonyl stresses calculated by the two systems, a high correlation was observed. The proposed system could measure carbonyl stress and distinguish the urine of 
diabetic rats from that of healthy rats. The detection time of $1 \mathrm{~s}$ is more in line with the requirements of a sensor than those of HPLC.

\section{Conclusion}

In this study, the fluorescence values measured on the standard calibration curve of methylglyoxal with concentrations ranging from 0 to $560 \mu \mathrm{g} / \mathrm{L}$ and different dilution ratios of rat urine showed a linear relationship. This shows the stability of the proposed simple fluorescence sensing system, which can be used for biological samples. Fourfold dilution achieved the most suitable urine dilution rate $\left(R^{2}=0.9879\right)$. Finally, we compared the fluorescence value measured by this system with the results of HPLC $\left(R^{2}=0.8132\right)$. This study preliminarily shows that the system can rapidly measure the carbonyl stress in urine with a small number of samples.

\section{Acknowledgments}

This work was financially supported by the National Taipei University of Technology- Taipei Medical University Joint Research Program (NTUT-TMU-103-12), and the Ministry of Science and Technology of Taiwan (109-2637-E-027-002-). The authors would like to thank Prof. Jen-Ai Lee at Taipei Medical University for her invaluable assistance.

\section{References}

1 K. M. Desai, T. J. Chang, H. Wang, A. Banigesh, A. Dhar, J. H. Liu, A. Untereiner, and L. Y. Wu: Can. J. Physiol. Pharmacol. 88 (2010) 273. https://doi.org/10.1139/Y10-001

2 T. Henle and T. Miyata: Adv. Renal Replace. Ther. 10 (2003) 321. https://doi.org/10.1053/j.arrt.2003.08.006

3 M. Kalousova, T. Zima, V. Tesar, S. Stipek, and S. Sulkova: Kidney Blood Pressure Res. 27 (2004) 18. https:// doi.org/10.1159/000075533

4 T. S. Omolaoye and S. S. du Plessis: Reprod. Toxicol. 93 (2020) 169. https://doi.org/10.1016/j. reprotox.2020.02.002

5 F. Heidari, S. Rabizadeh, A. Rajab, F. Heidari, M. Mouodi, H. Mirmiranpour, A. Esteghamati, and M. Nakhjavani: Life Sci. 260 (2020) 1. https://doi.org/10.1016/j.1fs.2020.118422

6 L. R. Bhat, S. Vedantham, U. M. Krishnan, and J. B. B. Rayappan: Biosens. Bioelectron. 133 (2019) 107. https://doi.org/10.1016/j.bios.2019.03.010

7 A. Espinosa-Mansilla, I. Duran-Meras, F. Canada Canada, and M. P. Marquez: Anal. Biochem. 371 (2007) 82. https://doi.org/10.1016/j.ab.2007.07.028

8 P. J. Thornalley: Drug Metab. Drug Interact. 23 (2008) 125. https://doi.org/10.1515/dmdi.2008.23.1-2.125

9 C. Nevin, L. McNeil, N. Ahmed, C. Murgatroyd, D. Brison, and M. Carroll: Sci. Rep. 8 (2018) 123. https://doi. org/10.1262/jrd.2020-150

10 M. Pastor-Belda, A. J. Fernandez-Garcia, N. Campillo, M. D. Perez-Carceles, M. Motas, M. HernandezCordoba, and P. Vinas: J. Chromatogr. A 1509 (2017) 43. https://doi.org/10.1016/j.chroma.2017.06.041

11 K. Dhananjayan, F. Irrgang, R. Raju, D. G. Harman, C. Moran, V. Srikanth, and G. Muncha: Anal. Biochem. 573 (2019) 51. https://doi.org/10.1016/j.ab.2019.02.014

12 A. G. Ojeda, K. Wrobel, A. R. C. Escobosa, M. E. Garay-Sevilla, and K. Wrobel: Anal. Biochem. 449 (2014) 52. https://doi.org/10.1016/j.ab.2013.12.014

13 A. Espinosa-Mansilla, I. Duran-Meras, and F. Salinas: Anal. Biochem. 255 (1998) 263. https://doi.org/10.1006/ abio.1997.2470

14 A. Sharma, D. Weber, J. Raupbach, T. C. Dakal, K. Fliessbach, A. Ramirez, T. Grune, and U. Wullner: Redox Biol. 34 (2020) 1. https://doi.org/10.1016/j.redox.2020.101546

15 C. M. Chen, S. M. Chen, P. J. Chien, and H. Y. Yu: J. Pharm. Biomed. Anal. 116 (2015) 150. https://doi. org/10.1016/j.jpba.2015.07.018 


\section{About the Authors}

Wei-Jan Huang received his B.S. degree from Taipei Medical University, Taiwan, in 1992 and his M.S. and Ph.D. degrees from National Taiwan University, Taiwan, in 1995 and 2002, respectively. Since 2013, he has been a professor at Taipei Medical University. His research interests are in the total synthesis and chemical modification of natural products and bioengineering. (wjhuang@tmu.edu.tw)

Yin-Tung Sun received his Ph.D. degree from the University of Cincinnati, USA. He is an associate professor at the Graduate Institute of Manufacturing Tech. in National Taipei University of Technology, Taiwan. His research interests include direct digital manufacturing, manufacturing system simulation, and simulators. (ytsun@mail.ntut.edu.tw)

Wei-Han Lee received his bachelor's degree in mechanical engineering from Chien-Hsin University of Technology, Zhongli, Taiwan, in 2018. He has been studying for a master's degree in mechanical engineering at National Taipei University of Technology since 2019. (kingman9697@gmail.com)

Po-Yeh Lin received his B.S. degree in 2016 and his M.S. degree in 2018 from Taipei Medical University. He has been a graduate student of the Ph.D. program at Taipei Medical University since 2018. His major is in pharmaceutical analysis. (poyeh0111@gmail.com)

Chien-Ming Chen received his Ph.D. degree in precision machinery engineering from the University of Tokyo, Tokyo, Japan, in 1999. Since 2013, he has been an associate professor at the Department of Electro-Optical Engineering, National Taipei University of Technology, Taiwan. His current research interests include biomedical optics, sensors, and nanophotonics. (cmchen@mail.ntut.edu.tw) 